\title{
Phenotypic Differences of Glu89Gln Genotype in ATTR Amyloidosis From Endemic Loci: Update From THAOS
}

\author{
Luca Gentile - Ivailo Tournev - Leslie Amass - Doug Chapman ·
}

Anna Mazzeo

Received: April 16, 2021 / Accepted: June 5, 2021 / Published online: June 19, 2021

(C) The Author(s) 2021

\section{ABSTRACT}

Introduction: Transthyretin amyloidosis (ATTR amyloidosis) is a progressive, clinically heterogeneous disease with spontaneous (wild-type) and hereditary (ATTRv) forms. The Glu89Gln variant is primarily associated with cardiomyopathy and prevalent in Italy and Bulgaria. The objective of this analysis was to better understand the profile of patients with ATTRv Glu89Gln amyloidosis in the Transthyretin Amyloidosis Outcomes Survey (THAOS).

Methods: THAOS is an ongoing, global, longitudinal, observational survey of patients with ATTR amyloidosis, including both inherited and wild-type disease, and asymptomatic

The THAOS investigators are shown in the Acknowledgements section.

L. Gentile $(\bowtie) \cdot$ A. Mazzeo

Department of Clinical and Experimental Medicine, University of Messina, Messina, Italy

e-mail: luca.gentile@unime.it

I. Tournev

Department of Neurology, Alexandrovska

University Hospital, Sofia Medical University, Sofia, Bulgaria

I. Tournev

Department of Cognitive Science and Psychology,

New Bulgarian University, Sofia, Bulgaria

L. Amass - D. Chapman

Pfizer Inc, New York, NY, USA carriers with mutations in the transthyretin gene. Demographic and clinical characteristics of all symptomatic patients with the ATTRv Glu89GIn variant enrolled in THAOS are described (data cutoff, January 6, 2020).

Results: There were 91 patients with ATTRv Glu89Gln amyloidosis with the majority from Bulgaria $(n=53)$ or Italy $(n=29)$. All patients were Caucasian and $50.5 \%$ were male. Patients from Bulgaria had a mean (standard deviation) age at enrollment of 57.1 (8.2) years, and duration of symptoms of 8.6 (9.6) years, compared with 54.8 (8.6) and 5.0 (4.1) years in Italy. In Bulgaria, $39.6 \%$ of patients were of a predominantly cardiac phenotype, $18.9 \%$ predominantly neurologic, and $41.5 \%$ mixed. In Italy, $3.4 \%$ of patients were predominantly cardiac, $62.1 \%$ predominantly neurologic, and $34.5 \%$ mixed.

Conclusions: The majority of patients with ATTRv Glu89Gln amyloidosis in THAOS are from Bulgaria or Italy. There were notable phenotypic differences, with the cardiac phenotype more common in Bulgaria and the neurologic phenotype more common in Italy. Over onethird of patients had a mixed phenotype, suggesting a potential role of multiple genetic and/ or environmental factors and the need for comprehensive assessment of all patients.

Trial Registration: ClinicalTrials.gov: NCT00628745. 
Keywords: ATTR; Bulgaria; Glu89Gln; Italy; Transthyretin amyloidosis

\section{Key Summary Points}

Why carry out this study?

Transthyretin amyloidosis (ATTR amyloidosis) is a progressive, clinically heterogeneous disease with spontaneous (wild-type) and hereditary (ATTRv) forms; the Glu89GIn variant is a rare mutation present in a small number of countries.

The Transthyretin Amyloidosis Outcomes Survey (THAOS) is the most comprehensive global registry of patients with ATTR amyloidosis and this analysis represents one of the largest cohorts of patients with ATTRv Glu89Gln amyloidosis.

\section{What was learned from the study?}

Patients with ATTRv Glu89Gln amyloidosis in THAOS are predominantly from Bulgaria and Italy.

The cardiac phenotype was more common in Bulgaria and the neurologic phenotype more common in Italy, although over one-third of the patients from these countries had a mixed phenotype.

The higher proportion of patients from these countries with a mixed phenotype than previously known suggests a potential role of multiple genetic and/or environmental factors and underscores the need for comprehensive assessment of all patients.

\section{DIGITAL FEATURES}

This article is published with digital features, including a summary slide, to facilitate understanding of the article. To view digital features

for this article go to https://doi.org/10.6084/ m9.figshare.14731293.

\section{INTRODUCTION}

Transthyretin amyloidosis (ATTR amyloidosis) is a progressive, devastating, and clinically heterogeneous disease with spontaneous (wildtype) and hereditary forms. Hereditary ATTR amyloidosis (ATTRv amyloidosis) is caused by mutations in the transthyretin gene (TTR), transmitted as an autosomal dominant trait [1]. The clinical picture is characterized by multisystem involvement, including the peripheral nerves, autonomic nervous system, and heart [2-4]. So far, over 140 different TTR mutations associated with ATTRv amyloidosis have been described [5, 6]. The Glu89Gln (p.Glu109Gln) variant seems to be particularly represented in Italy (especially Sicily) [7], and in Bulgaria where a "founder effect" was demonstrated [8] and was believed to be associated with predominantly cardiac involvement [9]. However, multidisciplinary examination of patients with this variant has revealed a mixed phenotype, including signs and symptoms of both cardiologic and neurologic disease [7]. Similarly, the Val30Met variant is associated predominantly with neurological symptoms, but may also include cardiac disease, particularly in the lateonset form [10]. Disease onset is around the age of 50 and usually presents with neuropathic symptoms. However, heart dysfunction with heart failure and sudden death are major clinical issues during the course of disease that typically manifest at later stages, after potentially remaining silent for many years after the onset of disease [11].

The Transthyretin Amyloidosis Outcomes Survey (THAOS) is an ongoing, global, longitudinal, observational survey of patients with ATTR amyloidosis, including both inherited and wild-type disease, and asymptomatic carriers with TTR mutations $[12,13]$. THAOS collects multinational, longitudinal data on the natural history of the disease from a large and diverse patient population to help inform the characterization of ATTR amyloidosis and improve disease diagnosis and patient management 
$[9,12-16]$. This analysis was conducted to gain a deeper understanding of the demographic and clinical profile of all symptomatic patients with ATTRv amyloidosis with the Glu89Gln variant enrolled in THAOS.

\section{METHODS}

The design and methodology of THAOS (ClinicalTrials.gov: NCT00628745) have been described previously in detail [13]. All patients enrolled in THAOS with the Glu89Gln variant at the data cutoff date were included in this analysis (data cutoff, January 6, 2020). All THAOS study sites received ethical or institutional review board approval prior to patient enrollment, and each patient provided written informed consent. The study followed the International Council for Harmonisation Good Pharmacoepidemiology Practice guidelines and the principles of the Declaration of Helsinki.

Demographic and clinical characteristics, TTR genotype, family history, and medical history were recorded at enrollment. Symptomatic patients included in the analysis were those with the Glu89Gin variant and $\geq 1$ symptom possibly or definitely related to ATTR amyloidosis. Neurologic progression was assessed using the Neuropathy Impairment Score in the Lower Limbs (NIS-LL; ranges from 0 to 88), with higher scores indicating greater impairment [17], and the modified polyneuropathy disability (mPND) score (ranges from I to IV), where I indicates sensory disturbance in lower limbs but preserved walking capacity; II indicates difficulties in walking but no need of a walking stick; IIIa indicates one stick or one crutch required for walking; IIIb indicates two sticks or two crutches required for walking; and IV indicates patient confined to a wheelchair or bed. Quality of life was assessed using the Karnofsky Performance Status score (ranges from 0 to 100\%), with lower scores indicating greater impairment in functional status. Cardiac function was assessed by echocardiogram and included leftventricular (LV) septal thickness and ejection fraction.

Patients were classified into one of three phenotypes, based on symptoms at enrollment in THAOS [9]. Patients with the predominantly cardiac phenotype were those: (1) with abnormal electrocardiogram (ECG) due to rhythm disturbance or heart failure or dyspnea; and (2) who did not have more than mild neurologic or gastrointestinal (GI) symptoms (excluding erectile dysfunction, constipation, and carpal tunnel syndrome). Patients with the predominantly neurologic phenotype were those: (1) with walking disability of any severity or other neurologic symptoms of any severity or GI symptoms (early satiety, nausea, vomiting, unintentional loss of weight, diarrhea, constipation, or fecal incontinence) of any severity; and (2) who did not have abnormal ECG due to rhythm disturbance or heart failure or dyspnea. Patients with the mixed phenotype were all remaining symptomatic patients who did not meet the criteria for either cardiac phenotype or neurologic phenotype.

\section{Statistical Analysis}

All data were analyzed descriptively. Data are presented as mean (standard deviation) and percentages unless stated otherwise.

\section{RESULTS}

Of the overall 111 patients with the Glu89Gln variant enrolled in THAOS, 91 were symptomatic and included in this analysis. The remaining 20 patients were asymptomatic gene carriers, of whom seven $(35.0 \%)$ were male. There was a larger proportion of asymptomatic gene carriers from Italy (13 of 42 total patients, $31.0 \%$ ) than from Bulgaria (four of 57 total patients, $7.0 \%)$. The majority of symptomatic patients were from Bulgaria $(n=53)$ or Italy $(n=29)$, with the other nine patients from Argentina $(n=1)$, Germany $(n=2)$, Spain $(n=4)$, and the USA $(n=2)$. 


\section{Demographic and General Clinical Data for Symptomatic Patients with ATTRv Glu89Gln Amyloidosis}

For patients with ATTRv Glu89Gln amyloidosis, the mean age at enrollment was 56.12 years, with an equal distribution of males $(50.5 \%)$ and females (49.5\%). Mean duration of symptoms was 7.30 years. Mean Karnofsky score was 79.31, while mean modified body mass index (mBMI) was 995.67 (Table 1). Patients from Bulgaria tended to be older at enrollment, to have a longer duration of ATTR amyloidosis symptoms, and to have a less severe Karnofsky score than patients from Italy.

\section{Phenotypic Characteristics}

The most frequent phenotype was mixed, reported in $38.5 \%$ of patients, followed by predominantly neurologic (35.2\%) and predominantly cardiac $(26.4 \%)$. Phenotypic distribution varied by country, with relatively greater proportions of the predominantly cardiac phenotype in Bulgaria and the predominantly neurologic phenotype in Italy (Fig. 1). The nine patients from other countries included three (33.3\%) with a mixed phenotype, four $(44.4 \%)$ predominantly neurologic, and two $(22.2 \%)$ predominantly cardiac.

\section{Neurological Findings}

In all patients with ATTRv Glu89Gln amyloidosis, mean (SD) NIS-LL score was 17.97 (15.76) and tended to be higher (greater impairment) in patients from Italy (19.35 [20.43]) compared with patients from Bulgaria (16.21 [12.77]). The three neurologic symptoms most frequently reported were neuropathic pain/paresthesia $(82.4 \%)$, tingling $(72.5 \%)$, and numbness (69.2\%) at distal lower and/or upper limbs. Among GI symptoms, the most common were unintentional weight loss $(48.3 \%)$ and diarrhea/constipation $(28.9 \%)$ (Table 2).

In patients with a neurologic or mixed phenotype, mPND score was I or II in over half of the patients (Table 3).

Table 1 Demographic and general clinical data of patients with symptomatic ATTRv Glu89Gln amyloidosis at enrollment in THAOS

\begin{tabular}{lllll}
\hline & $\begin{array}{l}\text { Overall } \\
(\boldsymbol{n}=\mathbf{9 1})\end{array}$ & $\begin{array}{l}\text { Bulgaria } \\
(\boldsymbol{n}=\mathbf{5 3})\end{array}$ & $\begin{array}{l}\text { Italy } \\
(\boldsymbol{n}=\mathbf{2 9})\end{array}$ & $\begin{array}{l}\text { Other } \\
\text { countries } \\
(\boldsymbol{n}=\mathbf{9})\end{array}$ \\
\hline Sex, $n$ (\%) & & & & \\
$\quad$ Male & $46(50.5)$ & $28(52.8)$ & $14(48.3)$ & $4(44.4)$ \\
Female & $45(49.5)$ & $25(47.2)$ & $15(51.7)$ & $5(55.6)$ \\
Race, Caucasian, \% & 100 & 100 & 100 & 100 \\
Age at entry into THAOS, years, mean (SD) & $56.12(8.23)$ & $57.11(8.20)$ & $54.82(8.63)$ & $54.41(6.88)$ \\
Duration of ATTRv amyloidosis symptoms, years, & $7.30(8.02)$ & $8.56(9.55)$ & $5.04(4.08)$ & $7.17(6.36)$ \\
$\quad$ mean (SD) & $79.31(15.91)$ & $83.02(12.18)$ & $74.44(20.25)$ & $70.00(15.28)$ \\
Karnofsky score, \%, mean (SD) & 995.67 & 988.51 & 1061.51 & 912.07 \\
mBMI, mean (SD) & $(261.43)$ & $(236.97)$ & $(365.22)$ & $(238.11)$ \\
\hline
\end{tabular}

ATTRv amyloidosis hereditary transthyretin amyloidosis, ATTRv Glu89Gln amyloidosis ATTRv amyloidosis associated with the Glu89Gln variant, mBMI modified body mass index, $S D$ standard deviation, THAOS Transthyretin Amyloidosis Outcomes Survey (data cutoff, January 6, 2020) 
Predominantly cardiac

Bulgaria $(n=53)$

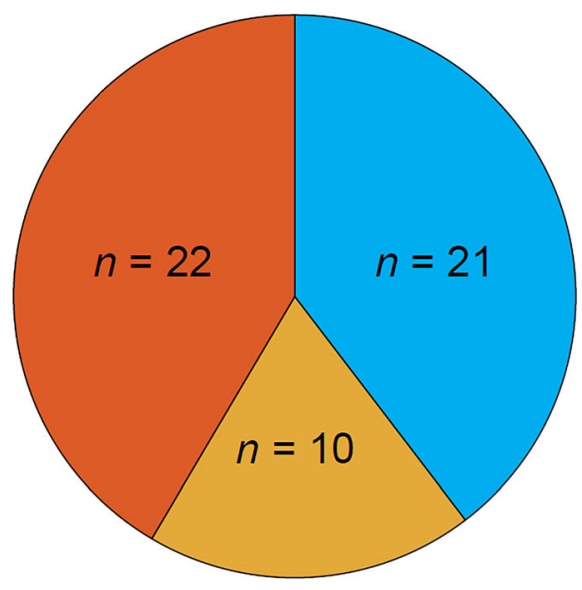

Fig. 1 Phenotypic categories of patients from Bulgaria and Italy with symptomatic ATTRv Glu89Gln amyloidosis at enrollment in THAOS (data cutoff, January 6, 2020). ATTRv Glu89Gln amyloidosis ATTRv amyloidosis

\section{Cardiac Findings}

In all patients with ATTRv Glu89Gln amyloidosis, symptoms related to heart failure were present in $55.6 \%$ of patients (Table 2). Palpitations (33.3\%), dyspnea (33.0\%), and rhythm disturbance $(24.2 \%)$ were also commonly reported.

In patients with a cardiac or mixed phenotype, mean LV septal thickness was $17.02 \mathrm{~mm}$ and mean ejection fraction was $56.04 \%$ (Table 4).

\section{DISCUSSION}

This THAOS analysis confirmed that patients with ATTRv Glu89Gln amyloidosis are found predominantly in Bulgaria (51.4\%) and Italy $(37.8 \%)$, with a minority of patients from other countries (10.8\%). A mixed phenotype was observed in at least one-third of these patients. Mean age of symptom onset was about 50 years, consistent with previously published data [7]. No notable differences between countries were

\section{Predominantly neurologic $\quad \square$ Mixed}

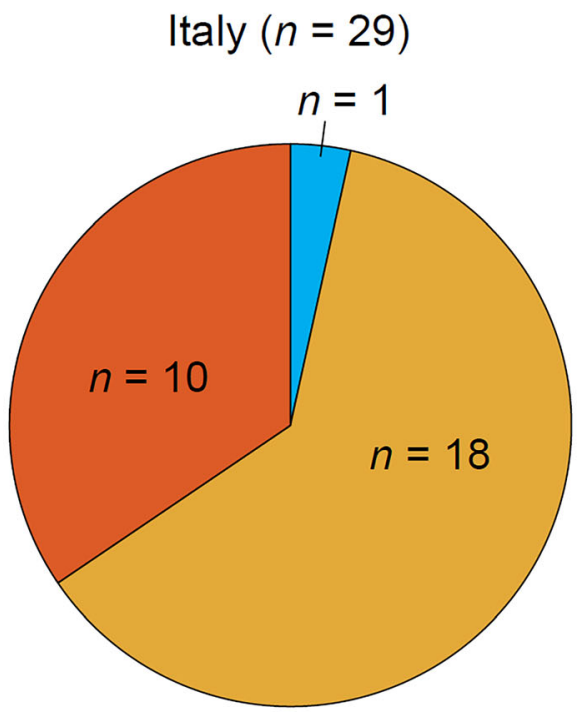

associated with the Glu89GIn variant; THAOS Transthyretin Amyloidosis Outcomes Survey

found in male/female ratio or in age at enrollment. Karnofsky scores showed that most patients were able to care for themselves, although the mean score was lower (indicating a greater impairment in functional status) in patients from Italy. mBMI was slightly higher in patients from Italy, who also presented with a notably shorter duration of symptoms than patients from Bulgaria.

Considering that Bulgaria and Italy have the highest known prevalence of the Glu89Gln variant, the difference in the distribution of phenotypes observed is interesting (Fig. 1). Patients from Bulgaria were more likely to have a cardiac phenotype, while most patients from Italy had a predominantly neurologic phenotype, although, as noted above, a mixed phenotype was observed in at least one-third of patients in both countries. There are three potential explanations for this difference. First, symptom duration differed across the countries, with patients from Bulgaria having a longer history of disease compared with patients from Italy. This difference in symptom duration could have allowed more time for cardiologic 
Table 2 ATTR amyloidosis-related symptoms reported in patients with ATTRv Glu89Gln amyloidosis at enrollment in THAOS

\begin{tabular}{lllll}
\hline & $\begin{array}{l}\text { Overall } \\
(\boldsymbol{n}=\mathbf{9 1})\end{array}$ & $\begin{array}{l}\text { Bulgaria } \\
(\boldsymbol{n}=\mathbf{5 3})\end{array}$ & $\begin{array}{l}\text { Italy } \\
(\boldsymbol{n}=\mathbf{2 9})\end{array}$ & $\begin{array}{l}\text { Other countries } \\
(\boldsymbol{n}=\mathbf{9})\end{array}$ \\
\hline $\begin{array}{l}\text { Neurologic symptoms, } n \text { (\%) } \\
\text { Neuropathic pain/paresthesia }\end{array}$ & $75(82.4)$ & $48(90.6)$ & $21(72.4)$ & $6(66.7)$ \\
Numbness & $63(69.2)$ & $31(58.5)$ & $24(82.8)$ & $8(88.9)$ \\
Temperature or pain insensitivity & $24(26.4)$ & $14(26.4)$ & $5(17.2)$ & $5(55.6)$ \\
Tingling & $66(72.5)$ & $42(79.2)$ & $17(58.6)$ & $7(77.8)$ \\
Gastrointestinal/autonomic symptoms, $n(\%)$ & & & \\
Diarrhea/constipation & $26(28.9)$ & $13(24.5)$ & $10(35.7)$ & $3(33.3)$ \\
Nausea & $16(18.0)$ & $11(20.8)$ & $5(18.5)$ & $0(0.0)$ \\
Early satiety & $29(33.0)$ & $23(43.4)$ & $4(15.4)$ & $2(22.2)$ \\
Unintentional weight loss & $43(48.3)$ & $32(60.4)$ & $7(25.9)$ & $4(44.4)$ \\
Cardiac symptoms, $n$ (\%) & & & \\
Palpitations & $30(33.3)$ & $26(49.1)$ & $1(3.6)$ & $3(33.3)$ \\
Rhythm disturbance & $22(24.2)$ & $16(30.2)$ & $5(17.2)$ & $1(11.1)$ \\
Dizziness & $38(41.8)$ & $25(47.2)$ & $7(24.1)$ & $6(66.7)$ \\
Heart failure & $50(55.6)$ & $40(75.5)$ & $6(21.4)$ & $4(44.4)$ \\
Dyspnea & $30(33.0)$ & $16(30.2)$ & $9(31.0)$ & $5(55.6)$ \\
Syncope & $20(22.0)$ & $13(24.5)$ & $4(13.8)$ & $3(33.3)$ \\
\hline
\end{tabular}

ATTRv Glu89Gln amyloidosis ATTRv amyloidosis associated with the Glu89Gln variant; THAOS Transthyretin Amyloidosis Outcomes Survey (data cutoff, January 6, 2020)

symptoms to emerge in patients from Bulgaria, resulting in a larger proportion of the mixed phenotype. Second, referring bias may have been a factor depending on whether the first diagnosis of ATTRv Glu89Gln amyloidosis was made by a neurologist or a cardiologist. Third, genetic factors other than the TTR mutation could influence phenotypic expression. The Bulgarian region endemic for the Glu89Gln variant is characterized by a relatively closed population and the presence of genetic forces such as inbreeding [8]. The same could be said for patients with the Glu89Gln variant from Italy, who have a clear common ancestor from a single city (Syracuse), with the Glu89Gln variant widely present in the nearby area $[7,11]$.
These findings on differences in patient phenotype by country are supported by the notable difference in NIS-LL and mPND scores. In patients from Italy, mean NIS-LL scores were more advanced and a greater proportion had an mPND score $>$ II (14.3 vs. $6.2 \%$ in patients from Bulgaria). No notable differences were observed in echocardiogram measures between the two countries, which may suggest that cardiomyopathy develops even in patients with predominantly neurological manifestations of ATTRv amyloidosis (such as those from Italy). Mean LV ejection fraction was not reduced in either country, which was as expected, as it is already known that dysfunction of the cardiac pump occurs only in the late stages of amyloid cardiomyopathy [18]. 
Table 3 Neurologic findings in patients with ATTRv Glu89Gln amyloidosis and predominantly neurologic or mixed phenotype

\begin{tabular}{lllll}
\hline & $\begin{array}{l}\text { Overall } \\
(\boldsymbol{n}=\mathbf{6 7})\end{array}$ & $\begin{array}{l}\text { Bulgaria } \\
(\boldsymbol{n}=\mathbf{3 2})\end{array}$ & $\begin{array}{l}\text { Italy } \\
(\boldsymbol{n}=\mathbf{2 8})\end{array}$ & $\begin{array}{l}\text { Other countries } \\
(\boldsymbol{n}=7)\end{array}$ \\
\hline mPND score, $n(\%)$ & & & & $0(0.0)$ \\
0 & $5(7.5)$ & $3(9.4)$ & $2(7.1)$ & $2(28.6)$ \\
I & $26(38.8)$ & $13(40.6)$ & $11(39.3)$ & $0(0.0)$ \\
II & $13(19.4)$ & $8(25.0)$ & $5(17.9)$ & $0(0.0)$ \\
IIIa & $2(3.0)$ & $1(3.1)$ & $1(3.6)$ & $1(14.3)$ \\
IIIb & $4(6.0)$ & $1(3.1)$ & $2(7.1)$ & $1(14.3)$ \\
IV & $2(3.0)$ & $0(0.0)$ & $1(3.6)$ & $3(42.9)$ \\
Missing & $15(22.4)$ & $6(18.8)$ & $6(21.4)$ & \\
\hline
\end{tabular}

ATTRv Glu89Gln amyloidosis ATTRv amyloidosis associated with the Glu89Gln variant, mPND modified peripheral neuropathy disability, THAOS Transthyretin Amyloidosis Outcomes Survey (data cutoff, January 6, 2020)

Table 4 Cardiac findings in patients with symptomatic ATTRv Glu89Gln amyloidosis and predominantly cardiac or mixed phenotype

\begin{tabular}{cllll}
\hline & $\begin{array}{l}\text { Overall } \\
(\boldsymbol{n}=\mathbf{5 9})\end{array}$ & $\begin{array}{l}\text { Bulgaria } \\
(\boldsymbol{n}=\mathbf{4 3})\end{array}$ & $\begin{array}{l}\text { Italy } \\
(\boldsymbol{n}=\mathbf{1 1})\end{array}$ & $\begin{array}{l}\text { Other countries } \\
(\boldsymbol{n}=\mathbf{5})\end{array}$ \\
\hline Echocardiogram & & & 7 & 3 \\
LV septal thickness, $n$ & 51 & 41 & $18.29(4.92)$ & $14.00(1.73)$ \\
mm, mean (SD) & $17.02(3.11)$ & $17.02(2.72)$ & 5 & 3 \\
LV diastolic thickness, $n$ & 49 & 41 & $41.40(5.77)$ & $42.33(2.52)$ \\
mm, mean (SD) & $43.69(5.40)$ & $44.07(5.52)$ & 6 & 3 \\
LV posterior wall, $n$ & 50 & 41 & $16.83(5.42)$ & $13.67(1.15)$ \\
mm, mean (SD) & $16.60(3.05)$ & $16.78(2.64)$ & 7 & 3 \\
LV ejection fraction, $n$ & 51 & 41 & $49.71(16.74)$ & $58.67(4.73)$ \\
\%, mean (SD) & $56.04(13.73)$ & $56.93(13.57)$ & & 2 \\
Cardiac biomarkers & & & 4 & $1326.65(1409.05)$ \\
NT-proBNP, $n$ & 9 & 3 & $7119.21(4328.82)$ & $7820.93(8658.64)$ \\
pg/ml, mean (SD) & $5143.85(6377.08)$ & & & \\
\hline
\end{tabular}

ATTRv Glu89Gln amyloidosis ATTRv amyloidosis associated with the Glu89Gln variant, $L V$ left ventricular, $N T$-proBNP $\mathrm{N}$-terminal pro-B-type natriuretic peptide, $S D$ standard deviation, THAOS Transthyretin Amyloidosis Outcomes Survey (data cutoff, January 6, 2020)

As Glu89Gln is a rare mutation present in a small number of countries, the number of patients who can be studied is limited. Differences in referral patterns between Bulgaria and
Italy may also have contributed to some of the differences seen in the predominant phenotype between the two countries. However, THAOS is the most comprehensive global registry of 
patients with ATTR amyloidosis and this analysis represents one of the largest cohorts of patients with ATTRv Glu89Gln amyloidosis. These data significantly add to the previously limited pool of knowledge on this genotype, emphasizing the heterogeneous nature of the disease with a substantial proportion of patients with Glu89Gln presenting with a mixed phenotype. This is consistent with the evolving understanding of many genotypes, with even patients with genotypes strongly associated with cardiac disease, such as the Val122Ile variant also showing some signs of neurological disease $[19,20]$.

\section{CONCLUSIONS}

The majority of patients with ATTRv Glu89Gln amyloidosis in THAOS were from Bulgaria and Italy, consistent with prior reports highlighting the concentration of this variant in these countries $[7,8,11]$. Differences in phenotypic manifestations of ATTRv Glu89Gln amyloidosis were also observed, with a predominantly cardiologic phenotype in Bulgaria and a predominantly neurologic phenotype in Italy. Importantly, a mixed phenotype was observed in over one-third of the patients in both countries, pointing to a need for comprehensive patient evaluation to ensure early diagnosis and patient management [21-23]. Taken together, these data also suggest the presence of distinct common ancestors in the respective areas, and the possible role of multiple genetic factors influencing disease manifestation of an otherwise monogenic disease.

\section{ACKNOWLEDGEMENTS}

We thank Dr. Jan Kiszko for his contributions to earlier versions of this work. We thank all THAOS patients and investigators for their important contributions to this study.

Additional THAOS investigators contributing to this analysis: Fabio Barroso, FLENI, Ciudad Autonoma de Buenos Aires, Argentina; Johan van Cleemput, Afdeling Klinische Cardiologie, O\&N I, Leuven, Belgium; Hartmut Schmidt,
Universitätsklinikum Münster - Transplant Hepatology, Münster, Germany; Burkhard Gess, University Hospital of RWTH Aachen, Aachen, Germany; Pablo Garcia Pavia, Hospital Universitario Puerta de Hierro, Majadahonda, Spain; José Luis Muñoz Blanco, Hospital Gregorio Marañón, Madrid, Spain; Claudio Rapezzi, Comitato Etico Indipendente dell Azienda, Bologna, Italy; Giuseppe Vita, AOU Policlinico G. Martino, Messina, Italy; Giampaolo Merlini, Centro per lo Studio e la Cura delle Amiloidosi Sistemiche, Pavia, Italy; Marco Luigetti, Fondazione Policlinico Gemelli - Universita Cattolica del Sacro Cuore, Roma, Italy; Yesim Parman, Istanbul University, Istanbul Faculty of Medicine, Department of Neurology, Istanbul, Turkey; Mathew Maurer, Columbia University Medical Center, New York, NY, USA; Samantha LoRusso, The Ohio State University College of Medicine, Columbus, OH, USA.

Funding. The THAOS registry and this analysis were sponsored by Pfizer. Medical writing support was provided by Caitlin Watson, PhD, of Engage Scientific Solutions, and funded by Pfizer, who also provided funding for the journal's Rapid Service Fee.

Authorship. All named authors meet the International Committee of Medical Journal Editors (ICMJE) criteria for authorship for this article, take responsibility for the integrity of the work as a whole, and have given their approval for this version to be published.

Author Contributions. All authors contributed to the design and conduct of the analysis; interpretation of the data; and preparation, review, and approval of the manuscript.

Disclosures. Luca Gentile reports travel grants from Kedrion and CSL Behring to attend scientific meetings. Ivailo Tournev reports sponsorship from Pfizer for participation in advisory boards, congresses, and conferences. Leslie Amass is a full-time employee of Pfizer and holds stock and/or stock options with Pfizer. Doug Chapman is a full-time employee of Pfizer and holds stock and/or stock options with Pfizer. Anna Mazzeo reports travel grants from 
Kedrion and CSL Behring to attend scientific meetings.

Compliance with Ethics Guidelines. All THAOS study sites received ethical or institutional review board approval prior to patient enrollment, and each patient provided written informed consent. The study followed the International Council for Harmonisation Good Pharmacoepidemiology Practice guidelines and the principles of the Declaration of Helsinki.

Data Availability. Pfizer provides secure access to anonymized patient-level data to qualified researchers in response to scientifically valid research proposals. Further details can be found at https://www.pfizer.com/science/ clinical-trials/trial-data-and-results.

Open Access. This article is licensed under a Creative Commons Attribution-NonCommercial 4.0 International License, which permits any non-commercial use, sharing, adaptation, distribution and reproduction in any medium or format, as long as you give appropriate credit to the original author(s) and the source, provide a link to the Creative Commons licence, and indicate if changes were made. The images or other third party material in this article are included in the article's Creative Commons licence, unless indicated otherwise in a credit line to the material. If material is not included in the article's Creative Commons licence and your intended use is not permitted by statutory regulation or exceeds the permitted use, you will need to obtain permission directly from the copyright holder. To view a copy of this licence, visit http://creativecommons.org/licenses/by$\mathrm{nc} / 4.0 /$.

\section{REFERENCES}

1. Planté-Bordeneuve V, Said G. Familial amyloid polyneuropathy. Lancet Neurol. 2011;10(12): 1086-97.

2. Adams D, Cauquil C, Labeyrie C. Familial amyloid polyneuropathy. Curr Opin Neurol. 2017;30(5): 481-9.
3. Russo M, Gentile L, Toscano A, Aguennouz M, Vita G, Mazzeo A. Advances in treatment of ATTRv amyloidosis: state of the art and future prospects. Brain Sci. 2020;10(12):952.

4. Ruberg FL, Maurer MS, Judge DP, Zeldenrust S, Skinner M, Kim AY, et al. Prospective evaluation of the morbidity and mortality of wild-type and V122I mutant transthyretin amyloid cardiomyopathy: the Transthyretin Amyloidosis Cardiac Study (TRACS). Am Heart J. 2012;164(2):222-8.

5. Nehashi T, Oikawa M, Amami K, Kanno Y, Yokokawa T, Misaka T, et al. Sporadic cardiac amyloidosis by amyloidogenic transthyretin V122I variant. Int Heart J. 2019;60(6):1441-3.

6. Rowczenio D, Wechalekar A. Mutations in hereditary amyloidosis (2015). http:// amyloidosismutations.com/mut-attr.php. Accessed Dec, 182020.

7. Mazzeo A, Russo M, Di Bella G, Minutoli F, Stancanelli C, Gentile L, et al. Transthyretin-related familial amyloid polyneuropathy (TTR-FAP): a single-center experience in Sicily, an Italian endemic area. J Neuromuscul Dis. 2015;2(s2):S39-48.

8. Kirov A, Sarafov S, Pavlova Z, Todorov T, Chamova $\mathrm{T}$, Gospodinova $\mathrm{M}$, et al. Founder effect of the Glu89Gln TTR mutation in the Bulgarian population. Amyloid. 2019;26(4):181-5.

9. Damy T, Kristen AV, Suhr OB, Maurer MS, PlantéBordeneuve $\mathrm{V}, \mathrm{Yu} \mathrm{CR}$, et al. Transthyretin cardiac amyloidosis in continental Western Europe: an insight through the Transthyretin Amyloidosis Outcomes Survey (THAOS). Eur Heart J. 2019. https://doi.org/10.1093/eurheartj/ehz173 (Epub ahead of print).

10. Finsterer J, Iglseder S, Wanschitz J, Topakian R, Löscher WN, Grisold W. Hereditary transthyretinrelated amyloidosis. Acta Neurol Scand. 2019;139(2):92-105.

11. Russo M, Obici L, Bartolomei I, Cappelli F, Luigetti M, Fenu S, et al. ATTRv amyloidosis Italian registry: clinical and epidemiological data. Amyloid. 2020;27(4):259-65.

12. Coelho T, Maurer MS, Suhr OB. THAOS-the Transthyretin Amyloidosis Outcomes Survey: initial report on clinical manifestations in patients with hereditary and wild-type transthyretin amyloidosis. Curr Med Res Opin. 2013;29(1):63-76.

13. Planté-Bordeneuve V, Suhr OB, Maurer MS, White B, Grogan DR, Coelho T. The Transthyretin Amyloidosis Outcomes Survey (THAOS) registry: design and methodology. Curr Med Res Opin. 2013;29(1): 77-84. 
14. González-Duarte A, Barroso F, Mundayat R, Shapiro B. Blood pressure and orthostatic hypotension as measures of autonomic dysfunction in patients from the Transthyretin Amyloidosis Outcomes Survey (THAOS). Auton Neurosci. 2019;222: 102590.

15. Maurer MS, Hanna M, Grogan M, Dispenzieri A, Witteles R, Drachman B, et al. Genotype and phenotype of transthyretin cardiac amyloidosis: THAOS (Transthyretin Amyloid Outcome Survey). J Am Coll Cardiol. 2016;68(2):161-72.

16. Kristen AV, Maurer MS, Rapezzi C, Mundayat R, Suhr OB, Damy T. Impact of genotype and phenotype on cardiac biomarkers in patients with transthyretin amyloidosis - report from the Transthyretin Amyloidosis Outcome Survey (THAOS). PLOS ONE. 2017;12(4):e0173086.

17. Coelho T, Vinik A, Vinik EJ, Tripp T, Packman J, Grogan DR. Clinical measures in transthyretin familial amyloid polyneuropathy. Muscle Nerve. 2017;55(3):323-32.

18. Griffin JM, Maurer MS. Transthyretin cardiac amyloidosis: a treatable form of heart failure with a preserved ejection fraction. Trends Cardiovasc Med. 2019;31(1):59-66.
19. Gentile L, Di Bella G, Minutoli F, Cucinotta F, Obici L, Mussinelli R, et al. Description of a large cohort of Caucasian patients with V122I ATTRv amyloidosis: neurological and cardiological features. J Peripher Nerv Syst. 2020;25(3):273-8.

20. Stancanelli C, Gentile L, Di Bella G, Minutoli F, Russo M, Vita G, et al. Phenotypic variability of TTR Val122Ile mutation: a Caucasian patient with axonal neuropathy and normal heart. Neurol Sci. 2017;38(3):525-6.

21. Adams D, Slama M. Hereditary transthyretin amyloidosis: current treatment. Curr Opin Neurol. 2020;33(5):553-61.

22. Maurer MS, Schwartz JH, Gundapaneni B, Elliott PM, Merlini G, Waddington-Cruz M, et al. Tafamidis treatment for patients with transthyretin amyloid cardiomyopathy. N Engl J Med. 2018;379(11): 1007-16.

23. Vita G, Vita GL, Stancanelli C, Gentile L, Russo M, Mazzeo A. Genetic neuromuscular disorders: living the era of a therapeutic revolution. Part 1: peripheral neuropathies. Neurol Sci. 2019;40(4):661-9. 